\title{
Introduction to Electronic Marketing Minitrack
}

\author{
Bruce D. Weinberg \\ Department of Marketing \\ Isenberg School of Management \\ University of Massachusetts, Amherst \\ Weinberg@Isenberg.umass.edu
}

\author{
Lenita M. Davis \\ Department of Marketing \\ College of Business \\ University of Arkansas at Little Rock \\ lmdavis@ualr.edu
}

\author{
Ajit Kambil \\ CFO Program \\ Deloitte LLP \\ akambil@deloitte.com
}

This minitrack continues in its fourteenth year, highlighting some of the most interesting studies in this area. We have accepted papers in two areas: 1) quantitative, empirical research with strong theoretical underpinnings, and 2) novel methods and approaches, including case studies and frameworks, for envisioning and creating effective forms of online marketing.

Antonia Köster, Christian Matt, and Thomas Hess, in "Does the Source Matter? How Referral Channels and Personal Communication Tools Affect Consumers' Referral Propensity," test whether referral propensity is higher for consumers acquired through social referrals vs. commonly used channels, such as search engines and online advertisements, finding that consumers acquired through social referrals are more likely to make a referral.

In "Will They Stay or Will They Go? An Examination of the Factors Influencing User Loyalty towards News Websites," Benedikt Berger, Andre Geimer, and Thomas Hess build a quality-valuesatisfaction-loyalty chain for news websites to test for antecedents of loyalty for news website. Results reveal that loyalty towards news websites depends on both utilitarian and hedonic value perceptions, which, in turn, are influenced by content quality.

Zhuolun Li, Robert Kauffman, and Bingtian Dai, in "Can I See Beyond What You See? Blending Machine Learning and Econometrics to Discover Household TV Viewing Preferences," employ natural language processing with explanatory modeling that uses econometrics as a basis for understanding TV viewing preferences at the household-level to form strategically useful insights about consumer behavior.

In "Usage of Social Media Systems in Customer Service Strategies," Alexander Rossmann, Tim Wilke, and Gerald Stei examine the efficacy of social media systems in customer complaint handling. They conceptualize and evaluate complaint handling quality across traditional and social media channels, and compare the impact of complaint handling quality on key performance outcomes.

In "Consumers' Sentiments and Popularity of Brand Posts in Social Media: The Moderating Role of Upvotes," Hamid Khobzi, Raymond Yiu Keung Lau, and Terence Chun-Ho Cheung, examine the effect of consumers' sentiments embedded in user-generatedcontent on the popularity of brand posts, and show that consumers' sentiments are associated with post popularity
In "Achieving more by saying less? On the Moderating Effect of Information Cues in Paid Search," Darius Schlangenotto, and Dennis Kundisch, analyze the impact of an information cue on user behavior in ad copies. Contrary to prevalent advice, results suggest that reducing the number of words in an ad is not always beneficial.

In "Impression Effect VS Click-through Effect: Mechanism Design of Online Advertising," Xiang Zou, Ruhai $\mathrm{Wu}$, and Weijun Zhong examine various mechanisms in search and display advertising by considering both ads' impression effect and click-through effect. They show a seesaw relationship between ads' two effects in search advertising. The advertiser whose advertisement has a strong click-through effect benefits relatively less from its impression effect.

Julia A. Jacobs, Stefan Klein, Christopher P. Holland, and Maximilian Benning, in "Online Search Behavior in the Air Travel Market: Reconsidering the Consideration Set and Customer Journey Concepts," employ an experiment, analyze the detailed search processes of air travelers, and formulate a revised model of the search funnel and suggests a different operationalization of the consideration set.

In "Membership Free Shipping Programs: Effect on Competition and Optimality of Member Fees," Zhong Wen, and Lihui Lin study how the membership free shipping (MFS) program affects firm competition and how to set the membership fee optimally. They find that MFS relaxes price competition, and that a firm which adopts MFS has a higher pricing band, In addition, they show how to set the optimal membership fee.

In "Complimentary Return-Freight Insurance as Quality Signals: A New Return Policy in E-commerce," Shidao Geng, Wenli Li, and Hao Chen build a conceptual economic model to explore what kind of online retailer should offer complimentary return-freight insurance. The main results show three separating equilibria, where only high quality online retailers will offer complimentary return-freight insurance. 